\title{
In vivo Simulation-Based Learning for Undergraduate Medical Students: Teaching and Assessment
}

\author{
Michail Sideris ${ }^{1, *}$ \\ Marios Nicolaides ${ }^{2} *$ \\ Jade Jagiello ${ }^{3}$ \\ Kathrine S Rallis ${ }^{2}$ \\ Elif Emin' \\ Efthymia Theodorou ${ }^{2}$ \\ John Gerrard Hanrahan ${ }^{4}$ \\ Rebecca Mallick ${ }^{5}$ \\ Funlayo Odejinmi ${ }^{6}$ \\ Nikolaos Lymperopoulos ${ }^{7}$ \\ Apostolos Papalois ${ }^{8}$ \\ George Tsoulfas (iD) 9 \\ 'Women's Health Research Unit, Queen \\ Mary University of London, London, UK; \\ ${ }^{2}$ Barts and The London School of Medicine \\ and Dentistry, Queen Mary University of \\ London, London, UK; ${ }^{3}$ University College \\ London Hospital, London, UK; \\ ${ }^{4}$ Addenbrooke's Hospital, Cambridge \\ University Hospitals, Cambridge, UK; \\ ${ }^{5}$ Princess Royal Hospital, Brighton and \\ Sussex University Hospitals NHS Trust, \\ Haywards Heath, UK; ${ }^{6}$ Whipps Cross \\ University Hospital, Barts Health NHS \\ Trust, London, UK; ${ }^{7}$ Guy's and St Thomas \\ Hospitals NHS Foundation Trust, London, \\ UK; ${ }^{8}$ Special Unit for Biomedical Research \\ and Education, Aristotle University of \\ Thessaloniki, Thessaloniki, Greece; \\ ${ }^{9}$ Department of Surgery, Aristotle \\ University of Thessaloniki, Thessaloniki, \\ Greece
}

*These authors contributed equally to this work

Correspondence: Michail Sideris Women's Health Research Unit, Queen Mary University of London, Yvonne Carter Building, 58 Turner Street, London, EI 2AB, UK

Email m.sideris@qmul.ac.uk

\begin{abstract}
An increasing emphasis on simulation has become evident in the last three decades following fundamental shifts in the medical profession. Simulation-based learning (SBL) is a wide term that encompasses several means for imitating a skill, attitude, or procedure to train personnel in a safe and adaptive environment. A classic example has been the use of live animal tissue, named in vivo SBL. We aimed to review all published evidence on in vivo SBL for undergraduate medical students; this includes both teaching concepts as well as focused assessment of students on those concepts. We performed a systematic review of published evidence on MEDLINE. We also incorporated evidence from a series of systematic reviews (eviCORE) focused on undergraduate education which have been outputs from our dedicated research network (eMERG). In vivo SBL has been shown to be valuable at undergraduate level and should be considered as a potential educational tool. Strict adherence to 3R (Reduce, Refine, Replace) principles in order to reduce animal tissue usage, should always be the basis of any curriculum. In vivo SBL could potentially grant an extra mile towards medical students' inspiration and aspiration to become safe surgeons; however, it should be optimised and supported by a well-designed curriculum which enhances learning via multi-level fidelity SBL.
\end{abstract}

Keywords: in vivo simulation-based learning, medical simulation, surgical education

\section{Simulation-Based Learning (SBL): A Modern Atlas of Medical Training}

From the birth of the first resuscitation mannequin in 1960, to the most recent development of advanced virtual reality trainers and robotic surgery simulators, medical simulation has evolved remarkably. ${ }^{1,2}$ This growth can not only be attributed to concurrent technological advancements, but also to the rise of modern medicine, including a growing medical knowledge, radical reformations in the healthcare system and the emergence of minimally invasive procedures. ${ }^{3}$ This modernisation brought key changes that transformed the profession and arguably drove simulation to take up the role of a modern Atlas of medical and surgical training.

Simulation in medicine can be defined as a means of imitating a skill, attitude, or procedure to train personnel in a safe and adaptive environment. It exists in various forms, including verbal training, simulated patients, hardware-based and computing devices, and animal tissue; live (in vivo), isolated tissue flaps (ex vivo) and cadaveric. In vivo simulation is arguably at the end of the spectrum as per its level of complexity. In this article, we primarily define in vivo simulation as any SBL module that exclusively incorporates the use of live animals; likewise, by the 
term ex vivo SBL we refer to the use of any harvested animal tissue. Some people argue that in vivo SBL can be multifactorial and may include the use of actors or other modalities, however in this article we focused and followed the aforementioned definition.

An increasing emphasis on simulation has become evident in the last three decades following fundamental shifts in the medical profession. ${ }^{4}$ These changes have affected hands-on specialties such as surgery even more. On the other hand, reduction in working hours and subsequent reduction in surgical training time have significantly led to reduction of training time. ${ }^{5,6}$ Furthermore, the introduction of techniques of a steeper learning curve and a growing emphasis on non-technical skills highlight even more the importance of a change in educational approaches. $^{7-9}$ Other driving forces include increased medical litigation, operating time pressures and rising student numbers (Figure 1). ${ }^{10-12}$

\section{Aim}

We aimed to summarise and critically present all the published evidence on in vivo Simulation-Based Learning (SBL) for undergraduate medical students; this includes primarily teaching concepts as well as focused assessment of students on those concepts.

\section{Methods}

We followed a three-stage approach:
- Initially, we performed a structured narrative review of the literature on MEDLINE using a simple keyword strategy, including a combination of terms such as simulation-based learning, in vivo, and undergraduate or medical students.

- Since authors are familiar with the topic, we integrated a core of evidence which had been previously published as part of their multifaceted surgical course. $^{13,14}$ This evidence was previously synthesised using a research concept named as "FOOVEL (Feedback studies, Objective Outcomes, Validated tool studies, Expert opinions, synthesis of the Literature)". ${ }^{15}$ This primarily involves a multi-modality evidence synthesis which includes a series of narrative ${ }^{16}$ and systematic reviews ${ }^{17}$ focused on SBL for undergraduates (eviCORE ${ }^{18-21}$ ), with focused original studies on in vivo SBL for undergraduates. $^{22-28}$ This work has been part of a dedicated research network. ${ }^{29}$

- Regarding assessment methods, we performed a subgroup adaptation and appraisal of a systematic review published on postgraduates' assessment tools, which summarises the current published evidence on assessment approaches. ${ }^{21}$

Finally, we performed a critical appraisal of the adaptation of in vivo SBL based on the new reality that has been brought on by COVID-19. A systematic review on the future of medical and surgical education, as well as an opinion letter from the authors, have been the basis for this section of the article. ${ }^{30,31}$

\section{$\downarrow$ TRAINING TIME}

个 LITIGATION
MINIMALLY INVASIVE SURGERY

OPERATING TIME PRESSURES $\uparrow$ MEDICAL ERRORS

个 STUDENT NUMBERS

\section{SIMULATION-BASED TRAINING}

\section{个 PRACTICAL AND NON-TECHNICAL SKILL PERFORMANCE}

\section{$\downarrow$ TRAINEE STRESS}

ASSESSMENT

EVALUATION $\uparrow$ PATIENT SAFETY

Figure I Driving factors for introduction of simulation-based training and its benefits. 


\section{Included Articles}

Our search yielded 6 articles, ${ }^{13,16,17,22,25,26}$ all of which represent part of our previous work as part of the ESMSC Marathon Course (esmsc.gr). ${ }^{13,14}$ Manual search in the references of the included articles yielded another 2 original studies using in vivo SBL, which derive from the authors' previous work..$^{23,24}$

Following the "FOOVEL" approach, we completed the essential core of evidence needed to draw conclusions as part of this article. This includes in total 25 citations. ${ }^{13-37}$

\section{Discussion}

\section{SBL for Undergraduates: Knowns and Unknowns}

SBL has long now been effectively used in medical schools both for teaching and assessment. It can be used for a wide array of skills, ranging from history taking to inserting a cannula or even suturing. Furthermore, the use of simulated patients in the Objective Structured Clinical Examination (OSCE) assessment method has proved catalytic in its establishment as the gold standard for clinical skill assessment. The benefits of using SBL in medical schools are so well-recognised that studies are shifting their focus away from evaluating its effectiveness as a discipline. In our experience, recent research is focusing on how SBL can be used to deliver more niche parts of the syllabus and assessing the effectiveness of higher fidelity simulators for medical students.

As discussed above, even though low fidelity simulators are extensively used, the utilisation of in vivo simulation in medical schools is scarce. A systematic review by Theodoulou et $\mathrm{al}^{17}$ identified several studies that used animal tissue simulation for medical student training or assessment, however, only 2 used live animal tissue (in vivo). ${ }^{14,38}$ The results were promising in all studies that have used SBL for undergraduates, including the two in vivo studies.

\section{In vivo vs Low Fidelity Simulation}

In Vivo SBL has an established role as an advanced postgraduate training modality. However, at the undergraduate stage, its utility is still debatable; the soft line of ethics legislation behind the use of animal tissue, but also excessive costs, and complex laboratory set up are reasons to argue against animal use for medical student training. Currently, the Helsinki declaration is the gold standard framework for animal use in European territory. The UK has yet to adopt this 2010 framework, and this seems unlikely given the recent Brexit. Technological advances and the widespread use of dry lab low-cost, lower fidelity modules as a safe alternative, have gained place in favour of animal use, especially at earlier stages of training. This justifies the lack of published evidence on the topic; hence, anyone could wonder why we would use in vivo modules for medical students.

\section{Comparison Between in vivo vs ex vivo Modules and in vivo vs Dry Lab ${ }^{25,26}$}

As part of our course, ${ }^{13}$ we performed 2 studies to identify the role, or equally the additional benefit, of animal use for student training. Both studies have a common ground; the 3R (Reduce, Refine, Replace) principles. These dictate that animal use is minimised and its primary role is to provide an extra adjunct. This refers mainly for the use of live animals where we achieved a ration of $1 \mathrm{pig} / 20$ students achieving good quality intense training.

Both studies identified no additional benefit in the use of live animals in terms of practical skill improvement as defined by the use of the standardised Direct Observation of Procedural Skills (DOPS) tools. Simply put, this means that the vast majority of skills-based training can be equally effective with the use of low fidelity modalities. More specifically, students achieved similar proficiency when using the dry lab laparoscopic simulator, ${ }^{25}$ same applies for basic dissection skills when comparing the use of live pig vs ex vivo animal tissues. ${ }^{26}$

So, what would be the rationale behind using live in vivo SBL? To authors' opinion, it provides excitement and has a dominant inspirational character, which results in enthusiasm to take learning to the extra mile and enhances the relationship between trainer and trainee. In an era where burnout and bailout are the recipe of failure, inspiration and excitement can be a boost to push the youngest part of the medical pyramid to strive for perfection. In addition, if those modalities are carefully fitted in a multifaceted curriculum, then their role becomes multidimensional, and they aspire multifactorial benefit.

On a separate note, whilst measuring the perception of the educational environment of our course, ${ }^{27}$ we concluded that limited use of live tissue results in a more positive perception, which surprisingly is predominantly expressed by younger medical students.

Therefore, we could argue that eventually, providing high-quality simulation teaching at the earliest stage, this 
can eventually result in highly motivated who would be serving the vision of achieving excellence in patients' care.

\section{Descriptive in vivo SBL Studies ${ }^{22-24,28}$}

Similar results were noted in 3 non-comparative studies which demonstrate incorporation of animal use as part of our course. Two of them ${ }^{23,24}$ primarily use ex vivo animal tissue which can arguably be used with less cost and ethical implications, and are focused on basic neurosurgical skills, as well as the measurement of dexterity in micro-suturing. A similar concept was applied when trying to measure dexterity and anxiety and their role in SBL performance using ex vivo tissues; ${ }^{22}$ although this study did not use live tissue, it can act as an example on how such modalities are perceived by students in a busy learning environment. Another study takes Interventional Radiology (IR) a step forward and shows how lower fidelity SBL can be matched and optimised by the use of live tissue for IR catheterisation using a C-arm. ${ }^{28}$

\section{Multifaceted, Mixed Fidelity Animal Model SBL: The Esmsc Marathon Course [Unique] Model "Where Evidence Meets Novelty"}

The ESMSC Marathon Course ${ }^{13,14}$ is a 3-day multifaceted international surgical course geared exclusively to medical students. Its curriculum is designed to continuously evolve and currently involves 50 training modules spread across all basic surgical specialties. The ESMSC concept is based on the combination of 2 primary learning pylons (skills and knowledge) which are expanded in 4 learning cores (tetra-core concept):

-Basic and Applied Surgical Science knowledge in the form of interactive workshops

-Technical and Non-technical skills; technical skills are incorporating a unique combination of targeted minimal live animal tissue use (high fidelity), with ex vivo and dry lab SBL (low fidelity)

ESMSC is a paradigm of how in vivo SBL can grant the extra mile in SBL at the undergraduate level. Intense preparation of students using knowledge workshops, and skills optimisation in a short period of time, in the right lab environment produces the right momentum to use live in vivo modules. In vivo SBL as part of the ESMSC course has a dominant inspirational character, and students thrive from enthusiasm during such modules, which acts as a domino and enhances learning retention in the vast majority of the remaining modules. Further to this, using a high fidelity live operating environment help consolidate other learning components, given that students can practice most of the skills taught in the remaining skills modules.

\section{ESMSC Next Generation Concept: IG4}

The predominant question related to the use of in vivo live animal SBL is how this can be optimised. In other words, what component of the ESMSC curriculum should this occupy, in order to optimise learning and equally respect the $3 \mathrm{R}$ principles. To answer this question, we focused on designing cutting edge research to develop a theoretical curriculum model to harmonise the training modules across the 4 learning cores. The first version of this effort was the Ci4R version ${ }^{13}$ (Cores Integrated for Research) which evolved into a completely novel harmonised model, the iG4 (integrated Generation 4$)^{36}$ curriculum which is one of the most complex curricula concepts to ever be described in the literature. The iG4 concept was presented to a panel of experts ${ }^{37}$ and piloted in an advanced simulation centre to bring the first results. ${ }^{15}$ The primary goal of this advanced concept is to optimise learning via multilayer coordination of the several training modules of this multifaceted course. Thus, the educational value of the small live in vivo simulation modules is tailored and adapted to the wider concept of the course, optimising and enhancing its educational value. The iG4 vision is to act as a virtual engine to develop and process tailored training modules which serve the learning approach of multifaceted surgical courses. From the iG4 research, a separate version was produced (omnigon iG4), which can act as an adaptable global blueprint when designing such complex courses. ${ }^{18}$

\section{SBL Pragmatic Performance Model}

An additional benefit from the iG4 concept is the introduction of a second-generation hexagon (6-pylon) pragmatic model which assesses the overall performance of the course. ${ }^{18}$ Aspired by Harvard Business School's "balanced scorecard model", the "hexagon model" assesses: how customers (stakeholders) see the course curriculum, what trainers and organisers should excel in (internal perspective), if we can continue to improve and create value (research and innovation), and how course organisers appear to shareholders (financial perspective, adaptability, and sustainability in a global setting). The ESMSC 
hexagon model is a fourth-generation concept (iG4) which grants an edge when optimising the course structure and subsequently the use of animals at the undergraduate level. It lends its "pragmatic" character to the scrutiny of the course from several angles creating a $360^{\circ}$ view of how to constantly adapt and optimise learning using any local facility setting.

\section{Challenges in SBL}

One can easily deem surgical simulation methods as being abstract and "fake", as they cannot replace the actual clinical environment. Indeed, low-fidelity simulations often lack important factors, such as stress and responsibility, demoting the authenticity of the training experience. $^{39}$ A rapid degradation in authenticity and a consequent lack of effort by the trainee can lead to acquirement of false traits and techniques. Furthermore, cheap surgical simulators typically belong to the lowfidelity category. ${ }^{4}$ This becomes particularly problematic in surgery, where the haptic effect is essential and, in its absence, training can lead to distortion of the manual forces one applies causing catastrophic effects. ${ }^{40}$ Moreover, the fact that less-resourceful training centres cannot acquire animal tissue produces an injustice in surgical education.

In the same way that surgical education has been inspired by the aviation sector and attained various simulation techniques, it only makes sense to also look at its errors. Recent examples are two fatal air-crashes of the Boeing $738 \mathrm{MAX}$, for which the company has blamed the simulation equipment for not being able to mimic several conditions that caused the crash. Likewise, faults and inaccuracies in surgical simulators must be detected and corrected at all costs.

\section{Teaching Assessment Models}

Objective and reflective assessments are integral to all stages of medical education. Structured feedback allows learners to identify their educational needs and gauge their progress, both individually and in relation to their peers. Individual assessment is also used to certify the attainment of certain competencies. Within postgraduate educational settings, a trainee preference for regular work-based assessments has been identified, ${ }^{41,42}$ rather than single performance snapshots. ${ }^{4,44}$ SBL offers a valuable opportunity to realistically recreate these work-based scenarios, such that trainees may develop their technical and nontechnical skillset.
The aim for a novel SBL assessment tool is to holistically assess students' clinical skills and provide them with meaningful objectives. The key components of applicable knowledge being basic science, applied science, nontechnical and technical skills.

Due to limited published evidence on undergraduate assessment methods, the authors performed a sub-group adaptation and appraisal of a published systematic review $^{21}$ of postgraduate surgical assessments. In postgraduate training, there are examples of assessment modalities for the aforementioned clinical skills. Within the systematic review, the identified studies were all crosssectional or cohort in design. A thematic analysis demonstrated the key assessment themes could be further divided into surgical skills, surgical procedures, autonomy, communication, history, clinical examination, theoretical knowledge, data, self-assessment and milestones. These themes were shared across a variety of specialities and countries. Within the available literature, surgical skills were the most commonly evaluated. There is good precedent for the use of active assessment in the improvement of surgical technique. Bohnen et al have published their system for improving procedural learning which provides real-time feedback during procedures. ${ }^{45}$

Overall, however, the systematic review demonstrated a lack of clear evidence base for current methods of assessment. There was also a lack of cohesion across specialities and across different geographical areas, despite the thematic similarity of assessments.

The recommendations for SBL assessment would be to implement evidence-based methods of evaluation. These assessments would benefit from multi-disciplinary and cross-speciality collaboration.

\section{Team Assessment Models (Team OSCES) $)^{33,34}$}

Medical practice is continuously evolving and all the more incorporating non-technical skills, predominantly advanced communicational skills. There is a shift from an individual-based approach towards a multidisciplinary team-based approach in everyday practice. Hence, we developed studies to optimise assessment of such skills, which focus on individuals' (students') assessment as part of a team. For this purpose, we used the iTOFT tool to evaluate students' performance as part of a trauma team, adapted for the local needs of the ESMSC course. ${ }^{34}$ We also compared several SBL fidelity modalities (hyper- 
realistic vs low fidelity team OSCE) ${ }^{33}$ using the TEAM tool. Both studies serve as an example of how nontechnical team-based assessment can supplement in vivo simulation as part of the same multifaceted curriculum and advance learning outcomes.

\section{COVID-19: Welcome to a New World in SBL}

The COVID-19 pandemic was first declared by the World Health Organisation on March 11, 2019. ${ }^{46}$ Medical education has been severely impacted by the restrictions which aim to reduce viral spread. As the world approaches the first anniversary of the pandemic, it too marks one year of disrupted learning for students who, will ultimately, become doctors. A published systematic review by the authors aimed to elucidate challenges and novel approaches to medical education during the COVID-19 pandemic. ${ }^{30}$ Seven subcategories of innovation were identified: tele-conferences, online learning, social media, telemedicine, simulation and virtual reality, assessments and remote anatomy learning.

Although the COVID-19 pandemic has clearly been disruptive and detrimental to learning, there are a number of innovations which have been rapidly developed. Some of these interventions may be better than the historical didactic format of education.

By augmenting the number of resources which can be used, individuals can adapt their ideal learning environment. COVID-19 has had a seismic effect on the current landscape of education, but at the same time consolidated the use of several innovations which predominantly derive from the use of novel technologies in education. Although these new circumstances arguably shift away from the use of in vivo SBL, the demand of skills-based education, especially in advanced postgraduate level is higher, due to a long cease of most operating time. This dictates a new reality where all the innovations introduced during the pandemic, merge effectively with the traditional animal model simulation creating the ultimate environment for learning. In simple terms, technological innovations can optimise the use of in vivo SBL as part of a dedicated educational curriculum.

\section{Limitations}

We acknowledge a series of limitations. This review is predominantly based on a structured review of the literature as well as the authors' expert opinion on the topic.
The evidence on in vivo SBL is limited and should be interpreted with caution and constructive criticism. Despite the complex synthesis algorithm of the literature (eviCORE) that acts as an umbrella review of the current published evidence on In-Vivo SBL, there might still be several references available which are non-peer-reviewed and outside the scope of this review.

\section{Conclusion}

In vivo SBL should be used with caution at the level of undergraduate students. Strict adherence to the $3 \mathrm{R}$ principles in order to reduce animal tissue usage, should always be the basis of a planned course. In vivo SBL has a place in granting an extra mile towards medical students' inspiration and aspiration to become safe surgeons; however, this should be optimised and supported by a well-designed curriculum which enhances learning via multi-level fidelity SBL. A classic example is the ESMSC Marathon Course which uses an adaptable multifaceted curriculum design to incorporate composite learning outcomes.

\section{Place of Study}

Women's Health Research Unit, Queen Mary University of London, Yvonne Carter Building, 58 Turner Street, London, E1 2AB, UK

\section{Ethical Approval}

Ethical approval was not required for this manuscript directly; ESMSC Marathon Course (esmsc.gr) was granted by the Department of Animal Studies and Relevant Affairs (Hellenic Republic, Perifereia Attikis, PATT) and met directive 63/2010, PD 56/April 2013.

The license reference number is 4857/15-09-2017, MS, AP et al. All procedures performed in studies involving human participants were in accordance with the ethical standards of the institutional and/or national research committee and with the 1964 Helsinki declaration and its later amendments or comparable ethical standards. All applicable international, national and/or institutional guidelines for the care and use of animals were followed.

\section{Author Contributions}

Michail Sideris is the primary author of this work, has led and conceived the core of the evidence used for this article, including the ESMSC course, the iG4 model as well as the hexagon assessment performance model. Marios Nicolaides has primarily drafted this article with MS, and they are equal contributors for this review work. 
George Tsoulfas is the senior author of study. MS and GT have conceived this article and are responsible for the views expressed and the critical revision of this work Jade Jagiello and Kathrine Rallis, John Hanrahan, Elif Iliria Emin, Efthymia Theodorou, have contributed to drafting sections of the articles which were reviewed by MS. Rebecca Mallick, Funlayo Odejimi, Nikolaos Lymperopoulos and Apostolos Papalois have offered input in critically reviewing this manuscript. AP has been the lead of the ESMSC Marathon course with MS. All authors made substantial contributions to conception and design, acquisition of data, or analysis and interpretation of data; took part in drafting the article or revising it critically for important intellectual content; agreed to submit to the current journal; gave final approval of the version to be published; and agree to be accountable for all aspects of the work.

\section{Disclosure}

MS/AP are the leads of the ESMSC course; GT has extensive contribution in the eMERG project. The views expressed on this manuscript reflect the interpretation of the authors towards the topic and are based on previous work of the eMERG collaboration. The authors report no other conflicts of interest in this work.

\section{References}

1. Rosen KR. The history of medical simulation. J Crit Care. 2008;23 (2):157-166.

2. Badash I, Burtt K, Solorzano CA, Carey JN. Innovations in surgery simulation: a review of past, current and future techniques. Ann Transl Med. 2016;4(23):453.

3. Antoniou SA, Antoniou GA, Antoniou AI, Granderath FA. Past, present, and future of minimally invasive abdominal surgery. JSLS 2015;19(3):e2015.00052.

4. Agha RA, Fowler AJ. The role and validity of surgical simulation. Int Surg. 2015;100(2):350-357.

5. Parsons BA, Blencowe NS, Hollowood AD, Grant JR. Surgical training: the impact of changes in curriculum and experience. J Surg Educ. 2011;68(1):44-51.

6. Rodrigues IA, Symes RJ, Turner S, Sinha A, Bowler G, Chan WH. Ophthalmic surgical training following modernising medical careers: regional variation in experience across the UK. BMJ Open. 2013;3 (5):e002578.

7. Berwick D. A promise to learn - a commitment to act: improving the safety of patients in England. London: Department of Health; 2013.

8. Leape LL. Error in medicine. JAMA. 1994;272(23):1851-1857.

9. Donaldson MS, Corrigan JM, Kohn LT. To Err is Human: Building a Safer Health System. Vol. 6. National Academies Press; 2000.

10. Windsor JA. Role of simulation in surgical education and training. ANZ J Surg. 2009;79(3):127-132.

11. Kneebone R. Simulation in surgical training: educational issues and practical implications. Med Edu. 2003;37(3):267-277.

12. Bradley P, Postlethwaite K. Simulation in clinical learning. Med Edu. 2003;37:1-5.
13. Sideris M, Hanrahan J, Tsoulfas G, et al. Developing a novel international undergraduate surgical masterclass during a financial crisis: our 4-year experience. Postgrad Med J. 2018;94(1111):263-269.

14. Sideris M, Papalois A, Tsoulfas G, et al. Developing an international combined applied surgical science and wet lab simulation course as an undergraduate teaching model. Biomed Res Int. 2015;2015:463987.

15. Sideris M, Nicolaides M, Theodoulou I, et al. Student views on a novel holistic surgical education curriculum (iG4): a multi-national survey in a changing landscape. In vivo. 2020;34 (3):1063-1069.

16. Sideris M, Papalois A, Theodoraki K, et al. Promoting undergraduate surgical education: current evidence and students' views on ESMSC international wet lab course. J Invest Surg. 2017;30(2):71-77.

17. Theodoulou I, Nicolaides M, Athanasiou T, Papalois A, Sideris M. Simulation-based learning strategies to teach undergraduate students basic surgical skills: a systematic review. J Surg Educ. 2018;75 (5):1374-1388.

18. Sideris M, Emin EI, Hanrahan JG, et al. ABC of surgical teaching: time to consider a global blueprint for Holistic education. $J$ Invest Surg. 2020:1-11. doi:10.1080/08941939.2020.1800870.

19. Emin EI, Ruhomauly Z, Theodoulou I, et al. Are interventional radiology and allied specialities neglected in undergraduate medical education? A systematic review. Ann Med Surg. 2019;40:22-30.

20. Emmanuel T, Nicolaides M, Theodoulou I, Yoong W, Lymperopoulos N, Sideris M. Suturing skills for medical students: a systematic review. In vivo. 2021;35(1):1-12.

21. Hanrahan JG, Sideris M, Pasha T, Dedeilia A, Papalois A, Papalois V. Postgraduate assessment approaches across surgical specialties: a systematic review of the published evidence. Acad Med. 2021;96 (2):285-295.

22. Dhaif F, Paparoidamis G, Sideris M, et al. The role of anxiety in simulation-based dexterity and overall performance: does it really matter? J Invest Surg. 2019;32(2):164-169.

23. Hanrahan J, Sideris M, Pasha T, et al. Hands train the brain-what is the role of hand tremor and anxiety in undergraduate microsurgical skills? Acta Neurochir. 2018;160(9):1673-1679.

24. Hanrahan J, Sideris M, Tsitsopoulos PP, et al. Increasing motivation and engagement in neurosurgery for medical students through practical simulation-based learning. Ann Med Surg. 2018;34:75-79.

25. Pantelidis P, Sideris M, Tsoulfas G, et al. Is in-vivo laparoscopic simulation learning a step forward in the undergraduate surgical education? Ann Med Surg. 2017;16:52-56.

26. Sideris M, Papalois A, Theodoraki K, et al. Introducing in vivo dissection modules for undergraduate level trainees: what is the actual benefit and how could we make it more efficient? Indian J Surg. 2018;80(1):68-76.

27. Sideris MC, Papalois AE, Athanasiou T, et al. Evaluating the educational environment of an international animal model-based wet lab course for undergraduate students. Ann Med Surg. 2016;12:8-17.

28. Theodoulou I, Louca C, Sideris M, et al. A prospective study integrating a curriculum of interventional radiology in undergraduate education: a tetra-core simulation model. CVIR Endovasc. 2020;3 (1): 12 .

29. Sideris M, Hanrahan J, Staikoglou N, et al. Optimizing engagement of undergraduate students in medical education research: the eMERG training network. Ann Med Surg. 2018;31:6-10.

30. Dedeilia A, Sotiropoulos MG, Hanrahan JG, Janga D, Dedeilias P, Sideris M. Medical and surgical education challenges and innovations in the COVID-19 era: a systematic review. In vivo. 2020;34(3 Suppl):1603-1611.

31. Sideris M, Hanrahan JG, Papalois V. COVID-19 and surgical education: every cloud has a silver lining. Ann Med Surg. 2020;58:20-21.

32. Nicolaides M, Cardillo L, Theodoulou I, et al. Developing a novel framework for non-technical skills learning strategies for undergraduates: a systematic review. Ann Med Surg. 2018;36:29-40. 
33. Nicolaides M, Theodorou E, Emin EI, et al. Team performance training for medical students: low vs high fidelity simulation. Ann Med Surg. 2020;55:308-315.

34. Nicolaides M, Theodorou E, Hanrahan JG, et al. Advancing medical students' non-technical skills in a group-based setting. J Invest Surg. 2021;34(1):39-43.

35. Pantelidis P, Staikoglou N, Paparoidamis G, et al. Medical students' satisfaction with the applied basic clinical seminar with scenarios for students, a novel simulation-based learning method in Greece. J Educ Eval Health Prof. 2016;13:13.

36. Sideris M, Papalois V, Athanasiou T, et al. A novel multi-faceted course blueprint to support outcome-based holistic surgical education: the integrated generation 4 model (iG4). In vivo. 2020;34 (2):503-509.

37. Theodoulou I, Sideris M, Lawal K, et al. Retrospective qualitative study evaluating the application of IG4 curriculum: an adaptable concept for holistic surgical education. BMJ Open. 2020;10(2): e033181.

38. Ferreira Galvão FH, Bacchella T, Cerqueira Machado M. Teaching intestinal transplantation in the rat for medical student. Microsurgery. 2007;27(4):277-281.

39. Aggarwal R, Mytton OT, Derbrew M, et al. Training and simulation for patient safety. BMJ Qual Saf. 2010;19(Suppl 2):i34-i43.

40. Chmarra MK, Dankelman J, van den Dobbelsteen JJ, Jansen FW. Force feedback and basic laparoscopic skills. Surg Endosc. 2008;22 (10):2140-2148.
41. Miller A, Archer J. Impact of workplace based assessment on doctors' education and performance: a systematic review. $B M J$. 2010;341:c5064.

42. Shalhoub J, Marshall DC, Ippolito K. Perspectives on procedure-based assessments: a thematic analysis of semistructured interviews with 10 UK surgical trainees. BMJ Open. 2017;7(3): e013417.

43. Khan KZ, Gaunt K, Ramachandran S, Pushkar P. The objective structured clinical examination (OSCE): AMEE guide no. 81. Part II: organisation \& administration. Med Teach. 2013;35(9):e14471463.

44. Bode C, Ugwu B, Donkor P. Viva voce in postgraduate surgical examinations in Anglophone West Africa. J West Afr Coll Surg. 2011;1(1):40-52.

45. Bohnen JD, George BC, Williams RG, et al. The feasibility of real-time intraoperative performance assessment with SIMPL (System for Improving and Measuring Procedural Learning): early experience from a multi-institutional trial. J Surg Educ. 2016;73(6): e118-e130.

46. World Health Organisation. Timeline: WHO's COVID-19 response; 2021.
Advances in Medical Education and Practice

\section{Publish your work in this journal}

Advances in Medical Education and Practice is an international, peerreviewed, open access journal that aims to present and publish research on Medical Education covering medical, dental, nursing and allied health care professional education. The journal covers undergraduate education, postgraduate training and continuing medical education

\section{Dovepress}

including emerging trends and innovative models linking education, research, and health care services. The manuscript management system is completely online and includes a very quick and fair peer-review system. Visit http://www.dovepress.com/testimonials.php to read real quotes from published authors. 